\title{
ISCAA 2010 PRAGUE
}

2nd SYMPOSIUM OF CLINICAL AND APPLIED ANATOMY ISCAA 2010 PRAGUE PROFESSOR JOSEF STINGL JUBILEE

July 9th-11th, 2010 Prague, Czech Republic http://www.iscaa2010prague.cz

Abstract submission deadline 21st March, 2010.

\section{Topics:}

1. Developmental Anatomy and Histology

2. Applied Molecular Biology and Genetics

3. Anatomy, Histology, Embryology, Variations, Malformations, Diseases

- Locomotor System

- Cardiovascular System

- Respiratory System

- Digestive System

- Urogenital System

- Endocrine System

- Nervous System

- Sense Organs

4. Dental Investigations

5. Radiological Investigations
6. Anthropology

7. Comparative Anatomy

8. History of Anatomy, Histology and Embryology

9. Varia

\section{Delegates:}

Basic scientists, clinical anatomists, physicians and clinical researchers involved in the diagnosis and treatment of internal, surgical, neurological, gyneacological diseases and disorders, radiologists, pediatricians, otorhinolaryngologists, pathologists, nutritionists, geneticists, molecular biologists, public health specialists, general practitioners, and all other healthcare professionals.

\section{Other Informations:}

2nd Congress of the ISCAA

Institute of Anatomy

Third Faculty of Medicine

Charles University in Prague

Ruská 87, 10000 Prague 10

Czech Republic, EU

Phone +420-267-102508

Fax +420-26-102504

E-mail: info@iscaa2010prague.cz 